ten sich keine Widersprüche $\mathrm{zu}$ dem wiederholt postulierten Reaktionsmechanismus der IES-Oxydation durch $\mathrm{Mn}^{3+}$-Ionen, die über ein PeroxydasePhenol-System oxydiert worden sind ${ }^{5,17,21}$. Die Peroxydaseaktivität der Erbsenextrakte ist hoch; Mangankonzentrationen von $10^{-5}-m$. bis $10^{-2}-m$. bewirken in dialysierten Extrakten bei $p_{\mathrm{H}} 3,5$ eine annähernd lineare Aktivitätssteigerung der IES-Oxydase; auch hohe Manganzusätze bis zu $10^{-1}-\mathrm{m}$. bewirken keine Hemmung der dialysierten Extrakte; die durch DÄTC induzierte Hemmung kann durch zunehmende Mangan-Zusätze sukzessiv aufgehoben werden. Trotz dieser weitgehenden Übereinstimmung können einige Daten nicht direkt über das vorgeschlagene Schema erklärt werden, so z. B. die Hemmung undialysierter Extrakte durch Mangankonzentrationen von $1,7 \cdot 10^{-4}$ - $\mathrm{m}$. bzw. $1,7 \cdot 10^{-3}$-m. (bei $p_{\mathrm{H}} 6,0$ ) oder die Förderung durch niedere DÄTC-Konzentrationen in Verbindung mit Manganionen. Keine Erklärung findet vorläufig der Aktivitätsverlust bei Verwendung von Acetat-, Phthalatund Succinatpuffer, der nicht unbedingt, wie die hemmende Wirkung von Citrat oder Pyrrophosphat, über Bildung von stabilen Mangankomplexen 5, 16, 22 gedeutet werden kann.

Der von uns immer wieder beobachtete zweigipfelige Verlauf der $p_{\mathrm{H}}$-Optimumkurve ohne Manganzusatz und die Verschiebung des Optimums durch Manganionen könnten einen Ausgangspunkt zur differenzierten Analyse des Reaktionsmechanimus darstellen. In diesem Zusammenhang muß eine Beobachtung von Maclachlan und $\mathrm{W}_{\text {AYGood }}{ }^{17} \mathrm{er}$ wähnt werden, die über eine Erhöhung des $R Q \mathrm{im}$ sauren Bereich berichten. Es scheint, daß mit sinkendem $p_{\mathrm{H}^{-}}$Wert die Decarboxylierungs-Reaktion, die schon normalerweise der Oxydation vorangeht ${ }^{17}$, gegenüber der letzteren begünstigt wird. $\mathrm{Ob}$ die von uns beobachteten Gipfel der $p_{\mathrm{H}}$-Wirkungskurve einem dieser Reaktionsschritte zugehören, müssen differenziertere Untersuchungen klären.

Die Untersuchungen wurden mit Hilfe der D e u tschen Forschung semeinschaft durchgeführt, der wir hiermit danken.

Der A s grow Export Corporation, Milford, Connecticut, USA, die uns freundlicherweise das Erbsensaatgut zur Verfügung stellte, sprechen wir ebenfalls unseren Dank aus.

\title{
Die strukturellen Beziehungen zwischen pflanzlichen und tierischen Chondriosomen
}

\author{
Von E. Heitz \\ Aus dem Max-Planck-Institut für Biologie, Tübingen \\ (Z. Naturforschg. 12 b, 576-578 [1957]; eingegangen am 15. Mai 1957)
}

\begin{abstract}
Die Chondriosomen des Lebermooses Aneura pinguis und der Monokotyledone Zea Mais besitzen eine doppelte Membran und im Inneren röhrchenartige (bzw. blasenartige) Strukturen. Diese stehen bei beiden Pflanzen, durch eine schmale Einstülpung der inneren Membran in den Chondriosomen-Raum hinein, mit der Chondriosomen-Membran in Verbindung. Es wird die Ansicht vertreten, daß dieser Einstülpung (dem Sinn des Wortes entsprechend) ein Vorgang zugrunde liegen muß und es sich nicht lediglich um eine örtliche Beziehung (im Sinne von Sıöstrand) handelt.

Auf die Strukturhomologie von pflanzlichen und tierischen Chondriosomen wird nachdrücklich hingewiesen. Sie bestehen nicht nur in dem Vorhandensein einer das Chondriosom umgebenden äußeren und inneren Membran, sondern auch im Zustandekommen der inneren (Kulissen, Cristae) durch flächenhafte und (Röhrchen, Tubuli) durch räumliche, handschuhfinger-artige Einstülpungen der inneren Chondriosomen-Membran. Die Struktur-Homologie ist gesichert. Die Richtigkeit der Vorstellung von der Einstülpung der inneren das Chondriosom umgebenden Membran als Vorgang muß durch Untersuchung der Entwicklung junger Chondriosomen noch bewiesen werden.
\end{abstract}

Die vorliegende Arbeit schließt an eine kürzlich erschienene $^{1}$ an, in welcher (außer der Plastidenstruktur) auch die Struktur der Chondriosomen

1 E. Heitz, Z. Naturforschg. 12 b, 280 [1957]. erstmalig bei höheren Pflanzen eingehend behandelt wurde. Es hatte sich ergeben, daß die Chondriosomen im Wurzelmeristem von Zea Mais und Vicia Faba eine Membran besitzen und im Inneren röhrchenähnliche Gebilde enthalten. Über die Art der 
Verbindung dieser Tubuli wurde lediglich in einem Abb.-Text (Abb. 9) ${ }^{1}$ vermerkt, daß hier ein einzelner Tubulus als „Einstülpung der ChondriosomenMembran" erscheine; die Chondriosomen-Membran in den vorgelegten Abbildungen ist einheitlich. Es wurde aber in einer Anmerkung während der Drucklegung (l. c. S. 283) darauf hingewiesen, daß durch Aufnahmen mit dem Elmiskop I einwandfreie Belege für das Vorhandensein einer doppelten Membran erhalten wurden. Damit entstand die Frage nach dem genaueren Zusammenhang der Einstülpungen mit der als doppelt nachgewiesenen Chondriosomen-Membran. Die vorliegende Publikation bringt die photographischen Belege für das Vorhandensein dieser doppelten Chondriosomen-Membran bei einer einkeimblätterigen Pflanze und bei einem Lebermoos sowie über die Art des Zusammenhanges der Tubuli mit dieser doppelten Membran.

\section{Material und Methode}

Mit Erfolg benutzt wurden außer zwischen feuchten Filtrierpapier-Stücken oder in Sägemehl gekeimten und wachsenden Haupt- und Seitenwurzeln von Zea Mais, Thalli des Lebermooses Aneura pinguis. Diese wuchs auf 1,5-proz. Agar mit Prings h e i m scher Nährlösung (Erlenmeyerkölbchen) in algen- und pilzfreier oder auch absoluter Reinkultur, bei künstlichem Licht (Osram Bella Lux HNG 202, $40 \mathrm{~W}$. Entfernung der Kölbchen von der Röhre 5 bis $8 \mathrm{~cm}, 14 \mathrm{Stdn}$. Licht, $10 \mathrm{Stdn}$. Dunkelheit; Raumtemperatur $\pm 17^{\circ}$ ). Auch mit Wurzelspitzen einer Chlorophytum-Art wurden Resultate erhalten. Die Fixierung erfolgte wie üblich in $\mathrm{OsO}_{4} \mathrm{l}-2$ proz. in Veronal-acetat-Puffer $p_{\mathrm{H}} 7,2$. Die Einbettung wurde bei +2 bis +4 Grad während 2 , öfter 3 Stdn. in Methacrylat vorgenommen und nicht, wie bei der ersten Untersuchung ${ }^{1}$, in Polyester (jedoch ohne daß besondere Gründe dafür vorlagen). Aufnahmen erfolgten ausschließlich mit dem Elmiskop I von Siemens \& Halske.

Schnitte aus der Nähe der Scheitelregion von Aneura pinguis geben eindrucksvolle Übersichtsbilder. In Abb. ${ }^{*}$ sieht man den von 9 jungen Chloroplasten umgebenen Kern, ferner zwei der, für die

\footnotetext{
* Abb. 1-11 s. Tafel S. 578 a, b, c u. d.

2 A. J. Dalton u. M. D. Felix, Symposia Soc. exp. Biol. X, 148 [1957].
}

meisten Lebermoose charakteristischen Ölkörper. Drei Chondriosomen lassen sich ohne weiteres als solche erkennen. Bei stärkerer Vergrößerung (Abb. $2-8)$ läßt sich die doppelte, das Chondriosom umgebende Membran stellenweise (Abb. 2, Abb. 5-8) oder in ihrem gesamten Verlauf verfolgen (Abb. 3 und 4). Die Tubuli sind manchmal zu weiträumigen Blasen erweitert (vgl. vor allem Abb. 6-8), so daß man hierfür den Namen „vesiculae“ wählen könnte, vorausgesetzt, daß dieselben sich nicht als Artefakte (infolge von Fixierung oder während der Polymerisation des Methacrylates entstanden) erweisen sollten. Die Frage muß noch weiter verfolgt werden, denn auch solche bizarr eckige Formen wie in Abb. 3 (vgl. die Lagepause dazu, M 5 und 6) weisen vielleicht auf Artefaktbildung hin. Es sei immerhin auf eine Abbildung von Dalton und Felix hingewiesen $^{2}$, nach welcher bei der Protozoe Pelomyxa carolinensis ähnliche innere Chondriosomen-Strukturen vorhanden sein müssen. (Im Gegensatz zu anderen Protozoen wie z. B. Paramaecium und Vorticella.)

Die Abbildungen 2-8 geben nun auch den eindeutigen Nachweis, des direkten und ausschließlichen Zusammenhanges der Tubuli mit der inneren der beiden das Chondriosom umgebenden Membranen (vgl. Abb.-Texte und Lagepause zu Abb. 3).

Grundsätzlich dieselben Verhältnisse findet man bei den Chondriosomen im Wurzelmeristem von Zea Mais und auch einer Chlorophytum-Art. Die doppelte Membran ist in sämtlichen 6 Chondriosomen der Abb. 9, 10 und 11 über kleinere oder größere Strecken zu sehen (Abb. 9 und 10, jeweils das ganz links liegende Chondriosom). Den Zusammenhang der Tubulimembranen mit der inneren Chondriosomen-Membran allein belegen die mit Pfeilen bezeichneten Stellen auf Abb. 10 und 11 deutlich, etwas weniger deutlich die betreffende Stelle in 9.

Vergleicht man diese bei Aneura pinguis und Zea Mais gefundene Chondriosomen-Struktur mit der bei tierischen Chondriosomen festgestellten, so ergibt sich eine grundsätzliche Übereinstimmung. Daß bei den Chondriosomen der Tiere eine doppelte Membran vorhanden ist, wird heute nicht mehr bestritten, und durch die Untersuchungen von SEDAR und Porter ${ }^{3}$ an Paramecien, von Dalton und $\mathrm{FeLIX}^{2}$ an den Chondriosomen von Pelomyxa caro-

3 A. W. Sedar u. K. R. Porter, J. Biophys. Biochem. Cytol. I, 583 [1955]. 
linensis, an denjenigen der Ratte und Maus (Niere bzw. Leber) ${ }^{*}$, ferner von Low $^{4}$ an menschlichen Leukocyten ist sichergestellt, daß bei den verschiedensten Vertretern aus dem Tierreich die Oberflächen-Vergrößerung innerhalb des Chondriosom (gleichgültig, ob Cristae, Tubuli, Microvilli oder Vesiculae gebildet werden) durch Ausstülpung der inneren der beiden, überall vorhandenen, Chondriosomen-Membranen gegen den Innenraum des Chondriosom zustande kommt. (Als Belege wurden hier nur diejenigen Arbeiten angeführt, welchen restlos klare und eindeutige photographische Aufnahmen zugrun-

\section{1} de liegen.)

Es ist von einigem Interesse, daß auch die Oberflächen-Vergrößerung im Innenraum der Chondriosomen bei zwei Pflanzen (ganz verschiedener systematischer Stellung) auf die gleiche Weise vor sich geht. Man kann wohl erwarten, daß bei Untersuchung von Vertretern noch anderer Verwandtschaftskreise grundsätzlich dieselbe Entstehungsweise, bzw. vorsichtig ausgedrückt, dieselbe Struktur gefunden werden wird ${ }^{* *}$.

Auch Dalton und $\mathrm{F}_{\text {ELIX }}{ }^{2}$ betonen ausdrücklich, daß sie Einstülpung nicht nur als Beschreibung einer Struktur sondern als Vorgang der diese Struktur hervorgebracht haben muß, verstanden wissen wollen, wenn sie sagen: „the internal membranes at certain points are clearly continuous with and appear to be formed by an inward folding of the inner of the two limiting membranes". Als Illustration dafür (und nur als solche!) sei auf Abb. 3 (und die Lagepause) 0,3 hingewiesen. Derartige Stadien beginnender Einstülpung müßten sich in „jüngsten" noch aufzufindenden Chondriosomen nachweisen lassen, wenn die Auffassung als Vorgang (die ja eigentlich implicite in den Wortenden -ing und -ung enthalten ist) zu Recht besteht.

Wie dem auch sei, schon die aufgefundene Struktur-Gleichheit pflanzlicher und tierischer Chondrio-

\footnotetext{
* Sogar in Präparaten von Leberhomogenisaten in 30-proz. Glucose waren die doppelten Membranen gut erhalten und der Zusammenhang der inneren Membran mit den Tubuli zu erkennen (photographischer Beleg), auch wenn die Chondriosomen aufgequollen und die Grundsubstanz größtenteils nicht mehr sichtbar war. Es scheint, daß gerade fertig ausgebildete Lipoproteid-Membranen der Organellen der Zelle recht resistent gegenüber Behandlung mit Stoffen intra vitam sowie gegenüber Fixierung und Einbettung sind.
}
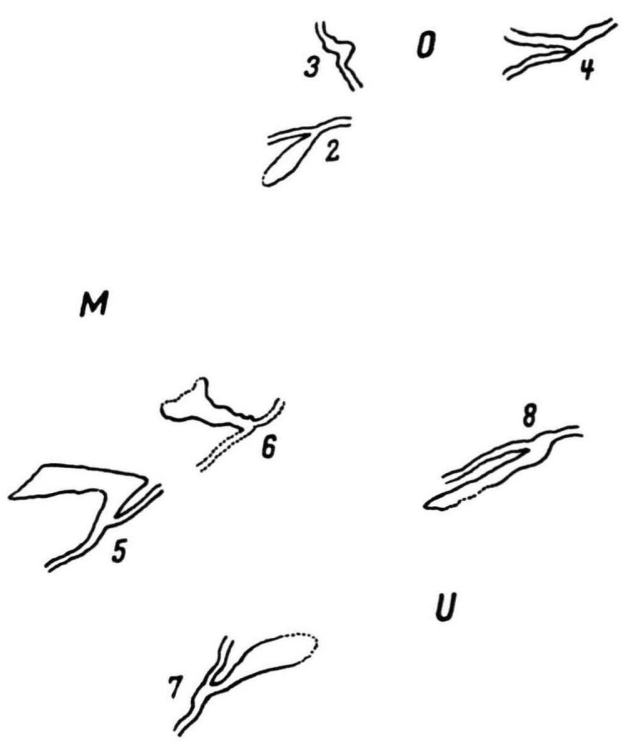

U

Abb. 3.

Lagepause von Abb. 3. In der Pause sind diejenigen Tubuli in derselben Lage und Größe eingetragen, wie sie in der Abbildung vorhanden sind, bei nicht ganz deutlichem Verlauf Membran punktiert. Zu dem oberen Chondriosom (0), gehören die Tubuli mit ihrem Ansatz an die innere Chondriosomen-Membran 4, zu dem mittleren (M) 1, 2, 5, 6; zu dem unteren (U) 7,8 .

somen ist in ähnlicher Weise erstaunlich, wie die der elektronenmikroskopischen Struktur von GeiBeln mit 2 im Zentrum und 9 an der Peripherie gelegenen Elementen durch das ganze Organismenreich hindurch (nur ganz vereinzelte Ausnahmen sind gefunden worden).

Die Deutsche Forschungsgemeinschaft stellte die zur Durchführung nötigen Mittel zur Verfügung. Dafür sei ihr bestens gedankt; ebenso Fräulein Dr. WeIchan und den technischen Assistentinnen Fräulein DörMER und Fräulein $\mathrm{W}_{\text {AGNER }}$ für tatkräftige und sorgfältige Hilfe. Dem Vorsteher des Hygienischen Instituts, Tübingen, Prof. Dr. Bader schulde ich großen Dank dafür, daß er ein Porter-Blum Mikrotom bereitwilligst zur Verfügung stellte.

4 F. N. Low, J. Bioph. Biochem. Cytol. 2, Supplement-Band, 337 [1956].

* Bei dem Pilz Allomyces macrogynus, wo durch Turian und Kellenberger [Exp. Cell Res. 11, 417 [19561) erstmals bei einer Pflanze eine doppelte ChondriosomenMembran und Cristae mitochondriales festgestellt wurden, findet sich noch kein Beleg für die Einstülpung der inneren Membran. Auch eine weitere Kopie, die mir Dr. Kellenberger liebenswürdiger Weise zur Verfügung stellte, ließ nichts davon erkennen. 

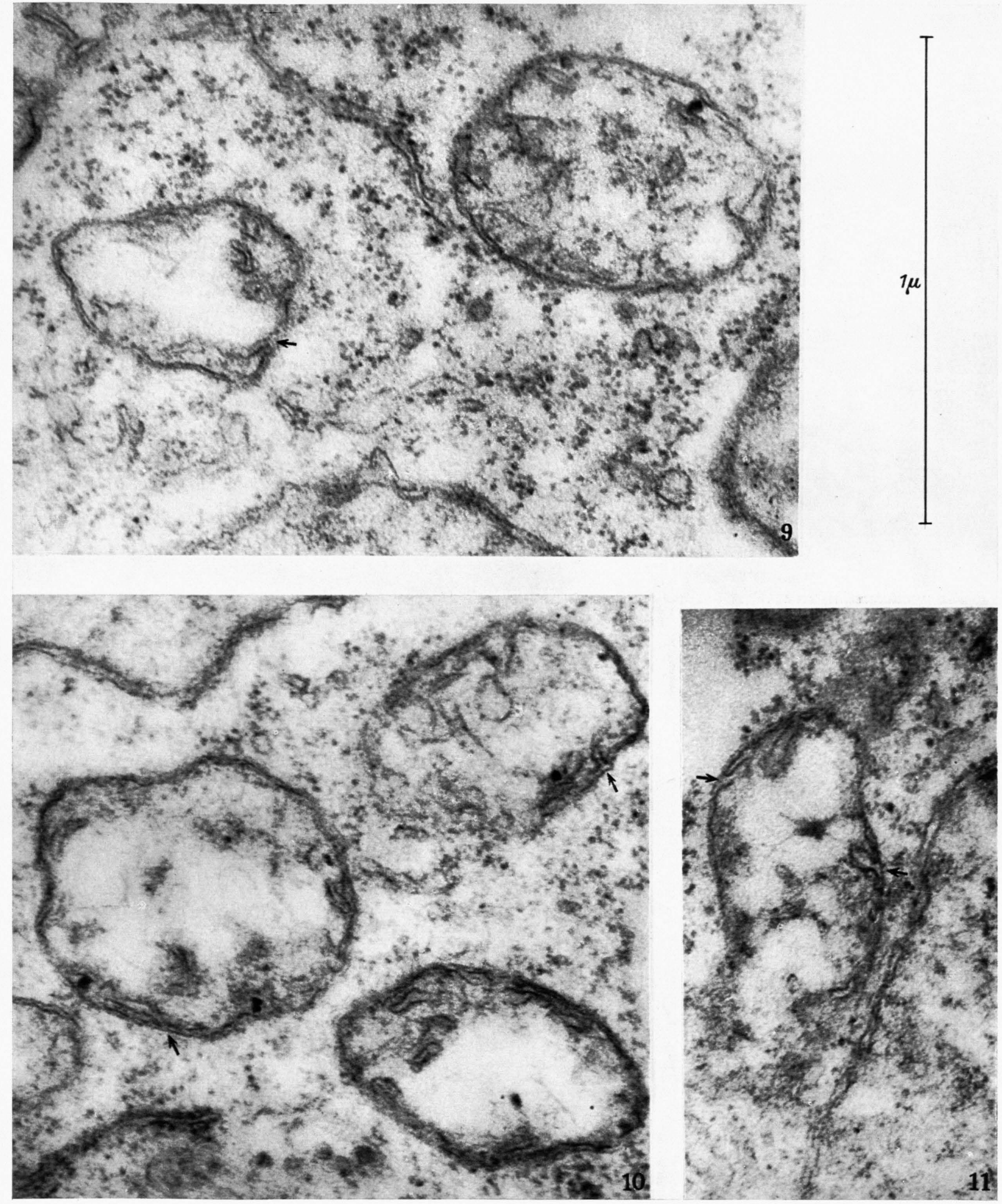
Abb. 1-8. Aneura pinguis, Thallus.

Abb. 1. Übersichtsbild über einen kleinen Teil einer embryonalen Zelle. In der Mitte der Zellkern mit einem großen und einem kleinen Nukleolus (ohne Membran, dichtkörnige Struktur) ; die doppelte Kernmembran ist in dem größeren Teil der Peripherie erkennbar. Rings um den Kern die lamellierten, z. T. Stärke enthaltenden (grau) Chloroplasten. Links und rechts der von mehreren Plasmodesmen durchzogenen Zellwand je ein von einer Membran umgebener Ölkörper; zwischen beiden, etwas oberhalb ein großes, hantelförmiges, rechts daneben ein kleines Chondriosom, und direkt links unterhalb anschließend eine kleine plasmatische Lamellenstruktur; links oben am Kern ein drittes Chondriosom. In der oberen rechten Bildecke und unterhalb der Mitte des rechten Bildrandes Plasma-Membranen, die Vakuolen abgrenzen. Vergr. 20000 -fach, el.-mikr. 10000 -fach. Abb. 2. Chondriosom aus (erwachsener) Thalluszelle; außer den blasenartigen Gebilden im Inneren erkennt man am rechten Ende eine doppelte Membran und ebenso ganz links oben und unten, wo das Zustandekommen der „Blase“ durch Einstülpung der inneren Membran nach dem Chondriosomen-Raum zu deutlich wird (Pfeil). Vergr. 70 000-fach, el.-mikr. 20000 -fach.
Abb. $3-5$, sowie Abb. 7 und Abb. 8. Vergr. 80 000-fach, el.mikr. 20000 -fach; Abb. 6. Vergr. 160000 -fach, el.-mikr. 40000 -fach. In jedem der 8 abgebildeten Chondriosomen, bzw. Chondriosomen-Teile, ist die doppelte Membran stückweise oder in ihrem ganzen Verlauf (Abb. 3 und 4) zu erkennen; ferner die Einstülpung allein der inneren Membran auf beiden Seiten (Abb. 3 bei 2, 5, 6, 7, 8 entsprechend der Abbildungspause; Abb. 6 und 8, Pfeile) oder wenigstens auf der einen Seite der jeweiligen „Blasen“-Basis (Abb. 3 bei 1, 4, entsprechend der Abbildungspause; Abb. 4, Abb. 5 und Abb. 7, Pfeile) .

Abb. 9-11. Zea Mais, Wurzelmeristem. Vergr. 80 000-fach, el.-mikr. 20000 -fach. Teile der doppelten Membran (100 bis $150 \AA$ breit) sind an allen 6 abgebildeten Chondriosomen sichtbar, in größerem Umfang bei dem rechten und vor allen Dingen bei dem linken Chondriosom in Abb. 9; außer den Tubuli (besonders in Abb. 10, rechtes unteres Chondriosom), an den durch 5 Pfeile bezeichneten Stellen auch die Einstülpung der inneren Membran deutlich; diese ist etwa $50 \AA$ breit. Im Plasma zahlreiche Granula, Durchmesser 100 bis 150 Å. In allen drei Abbildungen sind Stücke des Zellkerns mit doppelten Membranteilen vorhanden: in 9 unten Mitte, in 10 oben links, in 11 in drei Vierteln der rechten Bildhälfte. Unten links in 10 eine einzelne mit Granen besetzte plasmatische „granulöse Doppelmembran“.

Abbildungsunterschriften zu E. Heirz, Über Plasmastrukturen bei Antirrhinum majus und Zea Mais (S.579)

Abb. 1-4. Antirrhinum majus, in Nähe des Sproß-Vegetationspunktes.

Abb. 1. Links, Stück der Zellwand, von einem Plasmodesmos durchzogen; zwischen der Zellwand und nahe der Kernmembran (stückweise als doppelt erkennbar) 3 Gruppen von je 3, 5 und 3-4 Lamellen. Vergr. 48000 -fach, el.-mikr. 6000-fach.

Abb. 2. Oben dicht am Zellkern eine Gruppe von 3-4 Lamellen (unten rechts ein Chondriosom). Vergr. 32 000-fach, el.-mikr. $6000 \mathrm{fach}$.

Abb. 3. Links neben einem Plastid und direkt unterhalb der Kernmembran eine Gruppe von 3 Lamellen. Am unteren Bildrand Zellwand mit zwei Plasmodesmen. Aus demselben Schnitt wie Abb. 2. Vergr. 32 000-fach, el.-mikr. 6000-fach. Abb. 4. Ganz links eine Gruppe von 5 Lamellen und eine gleiche am rechten Bildrand. Die zweitoenannte Lamellengruppe liegt ganz nahe bei der doppelten Membran des Kernes (in der oberen rechten Hälfte am Bildrand), am unteren Bildrand die Zellwand mit zwei Plasmodesmen. Vergr. 30 000-fach, el.-mikr. 6000-fach. (Rechte untere Ecke der Abbildung ergänzt.)

Abb. 5-8. Zea Mais

Abb. 5. Embryonales Blatt, aus der Koleoptile eines eben gekeimten Samens herauspräpariert. In der Bildmitte zwei durch große Reserve-Stärkekörner gedehnte Plastiden, (Stärke infolge der Einbettung in Vinox weiß); der obere
Plastid, von welchem nur die Hälfte auf dem Bild vorhanden ist, von einer, fast im Verlauf der ganzen Peripherie erkennbaren Membran, umgeben; im unteren Plastiden ist diese nur auf der linken Seite erkennbar. In beiden Plastiden je eine größere etwa $1 \mu$ lange Stromalamelle (aus z. T. abgeplatteten Bläschen zusammengesetzt), im oberen Plastiden leicht, im unteren weniger leicht zu erkennen; außerdem sind je 3 Grana mit wenigen Lamellen vorhanden, die meistens quer getroffen; im oberen Plastid drückt ein kleines Stärkekorn durch sein Wachstum die angrenzenden Granalamellen etwas zusammen; rechts oben an dem kleinen Stärkekorn liegt ein schräg getroffenes Granum; ganz rechts unten im Bild ebenfalls ein etwas schräg getroffenes Granum unterhalb eines kleinen Stärkekorns. Zwischen der Zellwand (rechts) und dem großen Plastid liegt eine Gruppe von drei, etwa $2 \mu$ langen plasmatischen Doppellamellen; sie sind auf große Strecken mit dunklen kleinen Granula besetzt. Vergr. 47 250-fach, el.-mikr. 10500 -fach.

Abb. 6-8. Wurzelmeristem. Vergr. 80000 -fach, el.-mikr. 20000 -fach.

Abb. 6. Plasmastruktur von mehr oder weniger konzentrischen Ringen. Näheres vgl. Text.

Abb. 7. Gruppe von vier mit Granula besetzten Doppellamellen.

Abb. 8. Einzelne mit Granula verschiedener Größe besetzte Doppellamellen. Vergr. 80 000-fach, el.-mikr. 10 500-fach. 

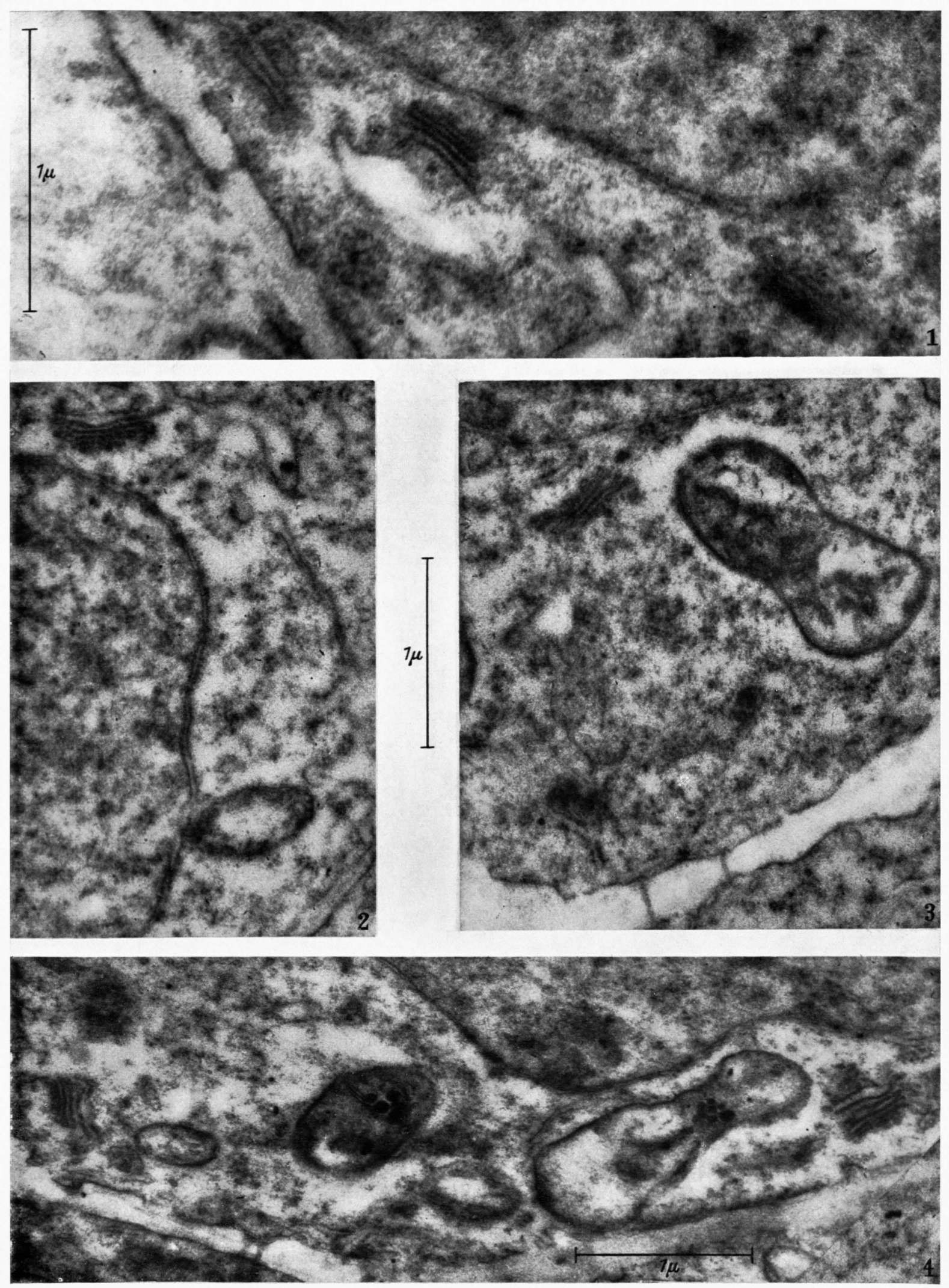


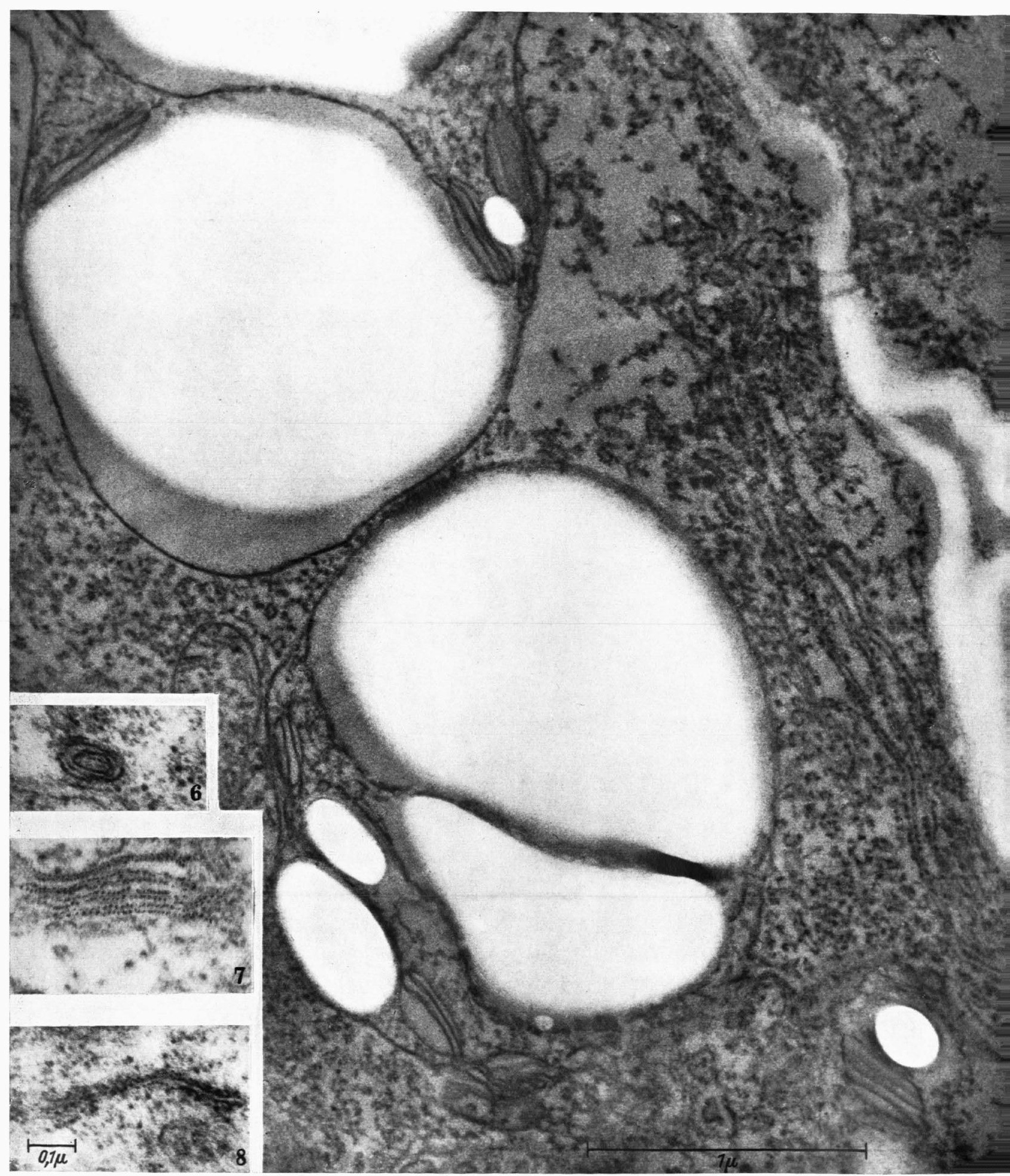

